\title{
Взаимодействие $\alpha$-частиц keV-энергий с молекулами глицил-лейцина
}

\author{
(C) А.А. Басалаев ${ }^{1}$, А.Г. Бузыкин ${ }^{2}$, В.В. Кузьмичев ${ }^{1}$, М.Н. Панов ${ }^{1}$, А.В. Петров ${ }^{3}$, \\ O.В. Смирнов ${ }^{1}$ \\ ${ }^{1}$ Физико-технический институт им. А.Ф. Иоффре РАН, Санкт-Петербург, Россия \\ ${ }^{2}$ Санкт-Петербургский политехнический университет Петра Великого, Санкт-Петербург, Россия \\ ${ }^{3}$ Институт химии Санкт-Петербургского государственного университета, Санкт-Петербург, Россия \\ E-mail: a.basalaev@mail.ioffe.ru
}

Поступило в Редакцию 26 февраля 2021 г.

В окончательной редакции 26 февраля 2021 г.

Принято к публикации 19 марта 2021г.

Изучены радиационные повреждения изолированных молекул глицил-лейцина $\left(\mathrm{C}_{8} \mathrm{H}_{16} \mathrm{~N}_{2} \mathrm{O}_{3}\right)$, вызванные взаимодействием с ионами $\mathrm{He}^{2+}$. Впервые получены относительные сечения основных процессов изменения зарядового состояния партнеров столкновений и относительные сечения процессов фрагментации однозарядных и двухзарядных молекулярных ионов, образующихся при однократных столкновениях молекул глицил-лейцина с ионами. Методом функционала плотности (DFT) рассчитана оптимизированная геометрия молекулы и однозарядного иона глицил-лейцина.

Ключевые слова: захват электронов, дипептиды, фрагментация, времяпролетная масс-спектрометрия.

DOI: 10.21883/PJTF.2021.12.51062.18746

Пептиды - важнейший класс биологических молекул, состоящих из аминокислотных остатков, связанных пептидной связью. Известно, что воздействие на пептиды ионизирующего излучения может вызывать структурные повреждения, приводящие к потере их функциональной активности. В частности, наблюдается возникновение разрывов полипептидной цепи, приводящих к появлению свободных амидных групп [1]. Радиационная устойчивость молекул может быть изучена при масс-спектрометрическом анализе молекулярных ионовфрагментов, образующихся в процессе однократных столкновений молекул в газовой фазе с ионизирующей частицей. Такой подход позволяет исключить из рассмотрения радиационно-химические процессы, происходящие при исследовании взаимодействия ионизирующего излучения с растворами или твердым телом. Исследования фотоэмиссионных спектров некоторых дипептидов, находящихся в газовой фазе, были выполнены в работах [2,3]. Масс-спектры ионов-фрагментов ряда дипептидов, образующихся при ионизации молекул электронным ударом, приведены в базе данных [4]. В первую очередь экспериментальные данные для изолированных молекул пептидов важны для проверки квантовохимических расчетов (см., например, [5]).

В настоящей работе были измерены относительные сечения взаимодействия ионов $\mathrm{He}^{2+}$ (энергия $E_{p}=4 \mathrm{keV} /$ a.m.u., скорость $V_{p}=0.41$ a.u.) с молекулами глицил-лейцина $\left(M=\mathrm{C}_{8} \mathrm{H}_{16} \mathrm{~N}_{2} \mathrm{O}_{3}\right)$ и изучена фрагментация образующихся молекулярных ионов $\{M\}^{n+}(n \geqslant 1)$

$$
\begin{aligned}
\mathrm{He}^{2+}+M & \rightarrow \mathrm{He}^{(2-s)+}+\{M\}^{n+}+(n-s) e^{-} \rightarrow \mathrm{He}^{(2-s)+} \\
& +\sum_{n} F r_{n}^{+}+\sum_{i} F r_{i}^{0}+(n-s) e^{-}
\end{aligned}
$$

где $\mathrm{He}^{(2-s)+}$ - налетающий ион в конечном зарядовом состоянии $(s=1,2), F r_{n}^{+}-$ионы-фрагменты (как правило, однозарядные), $F r_{i}^{0}-$ нейтральные фрагменты, $(n-s)$ - число свободных электронов, образующихся в процессах захвата с ионизацией. Для обозначения процессов использованы три числа, соответствующие начальному и конечному зарядовому состоянию налетающего иона и количеству образовавшихся заряженных фрагментов молекулы $-\{2(2-s) n\}$.

Исследования были выполнены с помощью „многостопового“ времяпролетного анализа зарядового и массового состава фрагментов молекулы, образующихся в процессах (1) [6]. Зарядовое состояние налетающего иона после взаимодействия одновременно определялось с помощью электростатического анализатора. Мишень изолированных молекул с плотностью, обеспечивающей однократность столкновений, создавалась сублимацией кристаллического образца при температуре $190 \pm 1^{\circ} \mathrm{C}$.

Относительные величины сечений элементарных процессов (1) определялись при анализе масс-спектров ионов-фрагментов, из которых вычитался фоновый массспектр, измеряемый при закрытом молекулярном пучке. Полученные после этой процедуры масс-спектры нормировались на полное количество налетающих ионов и количество вещества, прошедшего через область взаимодействия за время измерения спектра. Заряд промежуточного молекулярного иона $\{M\}^{n+}$ определялся по количеству одновременно образовавшихся ионовфрагментов и их зарядовому состоянию.

Для расчета оптимизированной геометрии молекулы и однозарядного иона глицил-лейцина (рис. 1) применялся модуль $\mathrm{Dmol}^{3}$ из программного пакета Materials Studio, в котором реализован метод функционала плотности (DFT). Были использованы функционал 

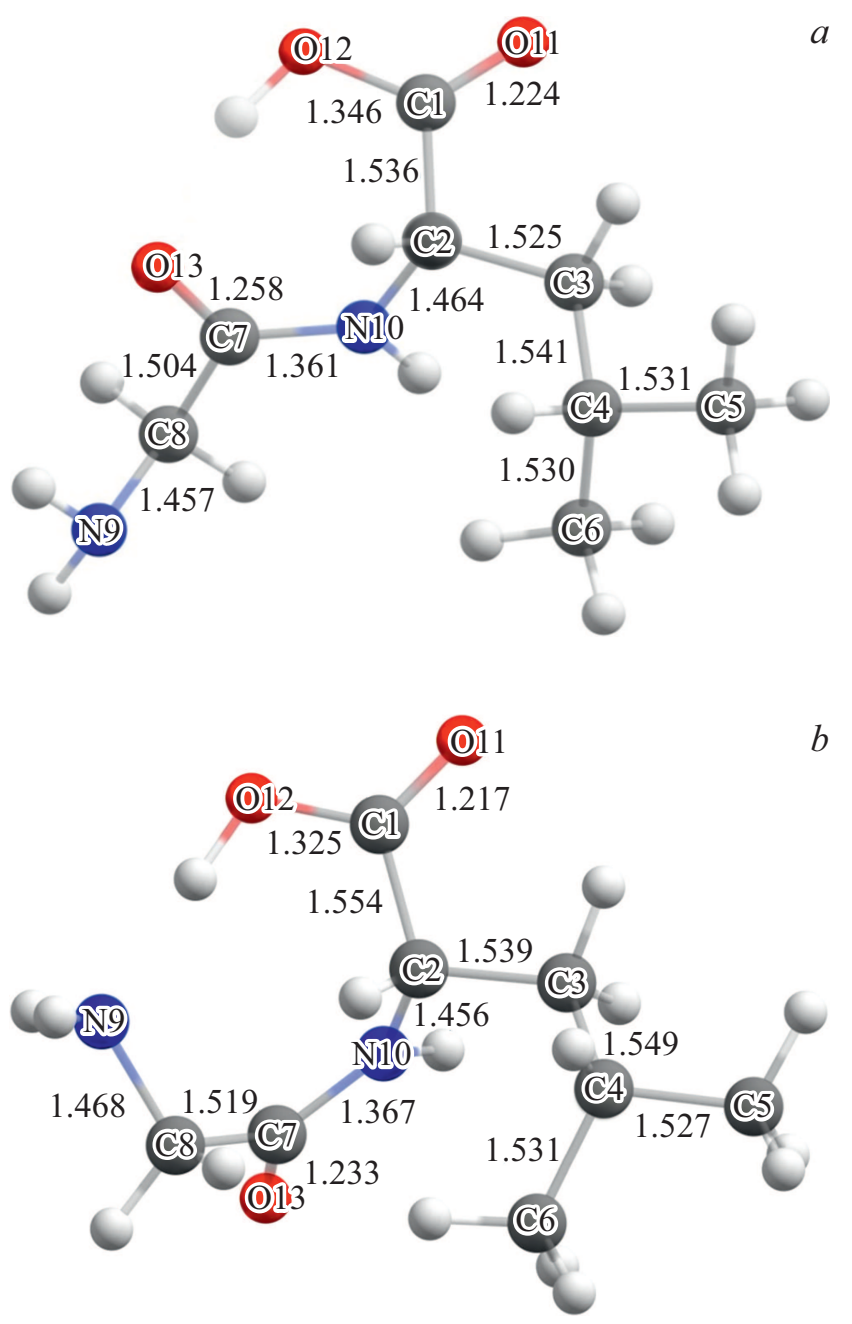

Рис. 1. Структурные формулы молекулы $(a)$ и однозарядного иона $(b)$ глицил-лейцина (расстояния между атомами указаны в $\AA$ ).

B3LYP и полноэлектронный базис с учетом поляризации DNP ver.4.4 [7,8].

Полученные после обработки масс-спектры ионовфрагментов, образующихся в процессах захвата одного и двух электронов, приведены на рис. 2. Интегрирование измеренных масс-спектров позволяет вычислить относительные сечения наблюдаемых элементарных процессов. Эти сечения приведены в табл. 1, в которой для полного сечения, равного сумме сечений всех измеренных элементарных процессов (1), принято $\sigma_{t o t}=100$ rel. un.

В табл. 2 приведены величины относительных интенсивностей основных ионов-фрагментов, образующихся в наиболее вероятных процессах $\{211\}$ и $\{202\}$. Идентификация ионов-фрагментов, приведенная в табл. 2, основана на том, что образование ионов массы $m$ происходит за счет разрыва минимального количества молекулярных связей и незначительной перегруппировки атомов между образующимися фрагментами. Приведенные в табл. 2 продукты фрагментации для процесса $\{211\}$ определяют $79 \%$, а для процесса $\{202\}-88 \%$ сечений процессов.
Таблица 1. Относительные сечения элементарных процессов $\{2(2-s) n\}$

\begin{tabular}{c|c}
\hline Процесс & $\begin{array}{c}\text { Относительное } \\
\text { сечение, rel. un. }\end{array}$ \\
\hline$\sigma_{\text {tot }}\{21 n\}$ & 100 \\
$\{211\}$ & $64.5 \pm 5$ \\
$\{212\}$ & $57.0 \pm 5$ \\
$\{213\}$ & $6.5 \pm 1$ \\
$\sum_{n}\{20 n\}$ & $1.0 \pm 0.3$ \\
$\{202\}$ & $35.5 \pm 2$ \\
$\{203\}$ & $25.5 \pm 2$ \\
$\{204\}$ & $8.7 \pm 0.7$ \\
& $1.3 \pm 0.3$
\end{tabular}

Из приведенных данных следует, что сечение образования недиссоциированного родительского иона мало, и это типично для большинства аминокислот [6,9]. Наиболее вероятными продуктами процесса одноэлектронного захвата являются фрагменты с массами $m=30$ и 114 a.m.u., что удовлетворительно согласуется с данными по фрагментации ионов глицил-лейцина, образующихся при ионизации электронами [4].

Фрагмент с массой 30 a.m.u. идентифицируется как ион $\mathrm{NH}_{2} \mathrm{CH}_{2}^{+}$, образующийся при простом разрыве связи С8-C7 (рис. 1). Как показывают расчеты геометрических параметров (рис. 1), в процессе ионизации наибольшее удлинение наблюдается для связей С8-C7 $(\Delta=0.015 \AA)$ и $\mathrm{C} 2-\mathrm{C} 1(\Delta=0.018 \AA)$. Отрыв нейтральной группы СООН от родительского молекулярного иона $\mathrm{M}^{+}$, сопровождаемый образованием иона с массой $m=143$ a.m.u., за счет разрыва связи С2-C1 экспери-

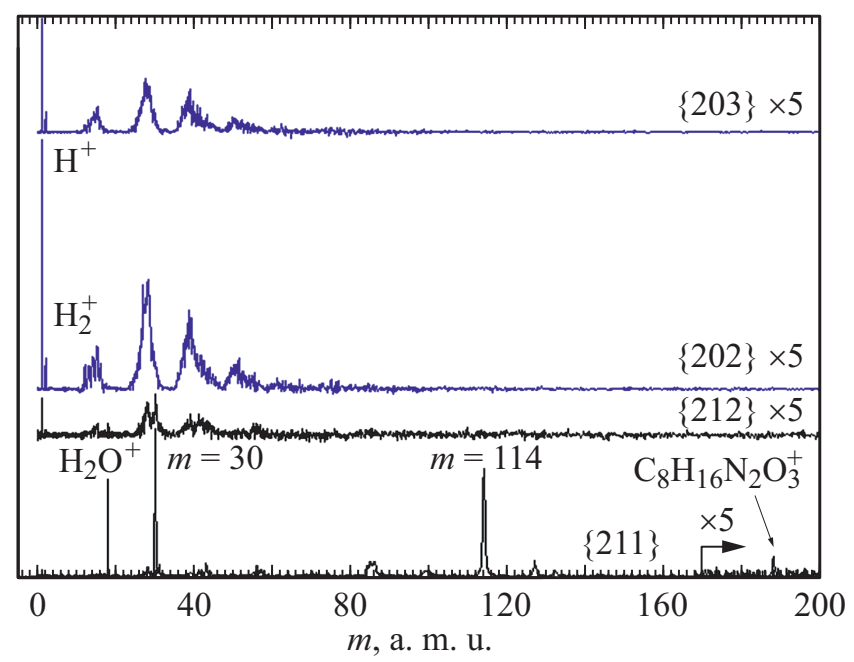

Рис. 2. Масс-спектры ионов-фрагментов, образующихся в процессах одноэлектронного захвата $\{211\}$, захвата с ионизацией $\{212\}$, двухэлектронного захвата $\{202\}$ и двухэлектронного захвата с ионизацией $\{203\}$. Спектры $\{212\},\{202\},\{203\}$ и часть спектра $\{211\}$, соответствующая массам 170-200 a.m.u., умножены на 5. 
Таблица 2. Интенсивность ионов, образующихся в процессах одноэлектронного $\{211\}$ и двухэлектронного \{202\} захвата (интенсивность фрагмента с массой 30 a.m.u. (процесс $\{211\}$ ) принята за 100)

\begin{tabular}{|c|c|c|c|c|c|}
\hline $\begin{array}{c}m, \\
\text { a.m.u. }\end{array}$ & Ион-фрагмент & $\{211\}$ & $\begin{array}{c}m, \\
\text { a.m.u. }\end{array}$ & Ион-фрагмент & $\{202\}$ \\
\hline 18 & $\mathrm{H}_{2} \mathrm{O}^{+}$ & 30.1 & 1 & $\mathrm{H}^{+}$ & 66.7 \\
\hline 28 & $\mathrm{HCNH}^{+}$ & 10.7 & 2 & $\mathrm{H}_{2}^{+}$ & 7.3 \\
\hline 30 & $\mathrm{NH}_{2} \mathrm{CH}_{2}^{+}$ & 100.0 & 12 & $\mathrm{C}^{+}$ & 7.1 \\
\hline 31 & $\mathrm{NH}_{2} \mathrm{CH}_{3}^{+}$ & 9.0 & 13 & $\mathrm{CH}^{+}$ & 9.3 \\
\hline 43 & $\mathrm{C}_{3} \mathrm{H}_{7}^{+}, \mathrm{C}_{2} \mathrm{H}_{5} \mathrm{~N}^{+}, \mathrm{C}_{2} \mathrm{H}_{3} \mathrm{O}^{+}$ & 11.0 & 14 & $\mathrm{CH}_{2}^{+}, \mathrm{N}^{+}$ & 16.8 \\
\hline 56 & $\mathrm{C}_{4} \mathrm{H}_{8}^{+}, \mathrm{C}_{3} \mathrm{H}_{6} \mathrm{~N}^{+}, \mathrm{C}_{3} \mathrm{H}_{4} \mathrm{O}^{+}$ & 9.8 & 15 & $\mathrm{CH}_{3}^{+}, \mathrm{NH}^{+}$ & 19.4 \\
\hline 84 & $\mathrm{C}_{3} \mathrm{H}_{4} \mathrm{~N}_{2} \mathrm{O}^{+}, \mathrm{C}_{4} \mathrm{H}_{4} \mathrm{O}_{2}^{+}$ & 9.1 & 16 & $\mathrm{NH}_{2}^{+}, \mathrm{O}^{+}$ & 14.4 \\
\hline 85 & $\mathrm{C}_{3} \mathrm{H}_{5} \mathrm{~N}_{2} \mathrm{O}^{+}, \mathrm{C}_{4} \mathrm{H}_{5} \mathrm{O}_{2}^{+}$ & 14.6 & 27 & $\mathrm{HCN}^{+}$ & 47.4 \\
\hline 86 & $\mathrm{C}_{3} \mathrm{H}_{6} \mathrm{~N}_{2} \mathrm{O}^{+}, \mathrm{C}_{4} \mathrm{H}_{6} \mathrm{O}_{2}^{+}$ & 13.8 & 28 & $\mathrm{HCNH}^{+}$ & 51.7 \\
\hline 114 & $\mathrm{C}_{4} \mathrm{H}_{6} \mathrm{~N}_{2} \mathrm{O}_{2}^{+}$ & 77.7 & 29 & $\mathrm{NH}_{2} \mathrm{CH}^{+}$ & 25.0 \\
\hline 115 & ${ }^{13} \mathrm{CC}_{3} \mathrm{H}_{6} \mathrm{~N}_{2} \mathrm{O}_{2}^{+}, \mathrm{C}_{4} \mathrm{H}_{7} \mathrm{~N}_{2} \mathrm{O}_{2}^{+}$ & 11.3 & 30 & $\mathrm{NH}_{2} \mathrm{CH}_{2}^{+}$ & 12.2 \\
\hline 127 & $\mathrm{C}_{7} \mathrm{H}_{13} \mathrm{NO}^{+}$ & 9.1 & 38 & $\mathrm{C}_{2} \mathrm{~N}^{+}, \mathrm{C}_{3} \mathrm{H}_{2}^{+}$ & 18.5 \\
\hline 188 & $\mathrm{C}_{8} \mathrm{H}_{16} \mathrm{~N}_{2} \mathrm{O}_{3}^{+}$ & 1.6 & 41 & $\mathrm{C}_{3} \mathrm{H}_{5}^{+}, \mathrm{C}_{2} \mathrm{H}_{3} \mathrm{~N}^{+}$ & 11.1 \\
\hline
\end{tabular}

ментально не наблюдается, хотя этот канал процесса фрагментации является для большинства алифатических аминокислот основным $[4,6,9]$. Отрыв иона $\mathrm{COOH}^{+}$ $\left(m=45\right.$ a.m.u.) от молекулярного иона $M^{+}$, как видно из рис. 2 , тоже маловероятен.

Фрагмент с массой 114 a.m.u. может быть идентифицирован как молекулярный ион диглицина $\mathrm{C}_{4} \mathrm{H}_{6} \mathrm{~N}_{2} \mathrm{O}_{2}^{+}$, высокая стабильность которого была показана в работе [10]. Образование этого иона сопровождается разрывом связи С2-C3 и формированием молекулы $\mathrm{C}_{4} \mathrm{H}_{8}$. У оставшегося иона-фрагмента, к которому происходит миграция атома водорода от аминокислотного остатка лейцина, формируется вторая пептидная связь с образованием свободной молекулы воды (2a), альтернативный канал реакции приводит к образованию иона воды и нейтральной молекулы диглицина $(2 \mathrm{~b})$ :

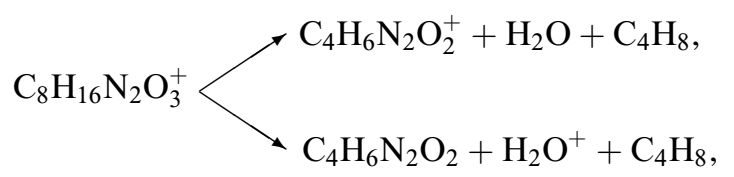

Как видно из рис. 2, в процессе $\{202\}$ образуются более легкие фрагменты, чем в процессе $\{212\}$. Средневзвешенная масса для процесса $\{211\}\langle m\rangle=63$ a.m.u., для процесса $\{212\}\langle m\rangle=36$ a.m.u. и для процесса $\{202\}$ $\langle m\rangle=23$ a.m.u. Заметное различие механизма фрагментации промежуточных двухзарядных ионов $M^{2+}$, образующихся в процессах $\{212\}$ и $\{202\}$, объясняется различием в энергиях их возбуждения, обусловленным тем, что в процессе $\{212\}$ энергия возбуждения частично уносится образовавшимся свободным электроном.

В заключение следует отметить, что процессы фрагментации многозарядных ионов объясняют наблюдение образования свободных амидных групп, возникающих за счет разрывов полипептидной цепи, при облучении пептидов рентгеновским излучением. Очевидно, что при взаимодействии с рентгеновским излучением наиболее вероятно образование именно ионов $M^{n+}$ с $n \geqslant 2$. При фрагментации однозарядных ионов разрыв пептидной связи маловероятен.

\section{Благодарности}

Расчеты проведены с использованием вычислительных ресурсов РЦ „Вычислительный центр СПбГУ“ (http://cc.spbu.ru).

\section{Финансирование работы}

Работа выполнена в рамках государственного задания (тема № 0040-2019-0023).

\section{Конфликт интересов}

Авторы заявляют, что у них нет конфликта интересов.

\section{Список литературы}

[1] Ю.Б. Кудряшов, Радиационная биофизика (ионизирующие излучения) (Физматлит, М., 2004), с. 153.

[2] V. Feyer, O. Plekan, R. Richter, M. Coreno, K.C. Prince, V. Carravetta, J. Phys. Chem. A, 113, 10726 (2009). https://doi.org/10.1021/jp906843j

[3] A.P. Wickrama Arachchilage, F. Wang, V. Feyer, O. Plekan, K.C. Prince, J. Chem. Phys., 133, 174319 (2012) DOI: $10.1063 / 1.3499740$

[4] NIST Chemistry WebBook [Электронный pecypc]. Режим доступа: https://webbook.nist.gov/chemistry/

[5] M.S. de Vries, P. Hobza, Ann. Rev. Phys. Chem., 58, 585 (2007). DOI: 10.1146/annurev.physchem.57.032905.104722

[6] О.В. Смирнов, А.А. Басалаев, В.М. Бойцов, С.Ю. Вязьмин, А.Л. Орбели, М.В. Дубина, ЖТФ, 84 (11), 121 (2014) DOI: $10.1134 / \mathrm{S} 1063784214110231$ 
[7] B. Delley, J. Chem. Phys., 92, 508 (1990). https://doi.org/10.1063/1.458452

[8] B. Delley, J. Chem. Phys., 113, 7756 (2000). https://doi.org/10.1063/1.1316015

[9] А.А. Басалаев, В.В. Кузьмичев, М.Н. Панов, О.В. Смирнов, Письма в ЖТФ, 43 (7), 63 (2017).

DOI: $10.21883 /$ PJTF.2017.07.44470.16531

[10] A.A. Basalaev, A.G. Buzykin, V.V. Kuz'michev, M.N. Panov, O.V. Smirnov, J. Phys.: Conf. Ser., 1400, 033017 (2019).

DOI: $10.1088 / 1742-6596 / 1400 / 3 / 033017$ 